\title{
Étude archéologique du môle nord-sud de Portus
}

Résultats de la campagne 2020

\section{Évelyne Bukowiecki, Ilaria Frumenti, Jean-Philippe Goiran et Milena} Mimmo

\section{(2) OpenEdition}

\section{Journals}

Édition électronique

URL : https://journals.openedition.org/baefe/3238

DOI : 10.4000/baefe.3238

ISSN : 2732-687X

Éditeur

ResEFE

Référence électronique

Évelyne Bukowiecki, Ilaria Frumenti, Jean-Philippe Goiran et Milena Mimmo, «Étude archéologique du môle nord-sud de Portus » [notice archéologique], Bulletin archéologique des Écoles françaises à l'étranger [En ligne], Italie, mis en ligne le 23 juillet 2021, consulté le 26 juillet 2021. URL : http:// journals.openedition.org/baefe/3238 ; DOI : https://doi.org/10.4000/baefe.3238

Ce document a été généré automatiquement le 26 juillet 2021.

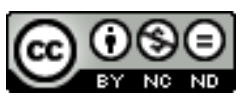

Le Bulletin archéologique des Écoles françaises à l'étranger est mise à disposition selon les termes de la Licence Creative Commons Attribution - Pas d'Utilisation Commerciale - Pas de Modification 4.0 International. 


\title{
Étude archéologique du môle nord-sud de Portus
}

Résultats de la campagne 2020

\author{
Évelyne Bukowiecki, Ilaria Frumenti, Jean-Philippe Goiran et Milena \\ Mimmo
}

\section{NOTE DE L'AUTEUR}

Date précise de l'opération : 8-30 septembre 2020

Autorité nationale présente : Parco Archeologico di Ostia Antica

Numéro de mission : $\mathrm{N}^{\circ}$ de concession de fouille : $1855 \mathrm{du}$ 05/05/2020

Composition de l'équipe de terrain : Évelyne Bukowiecki, École française de Rome ; Jorge Cocquyt, Sapienza Università di Roma ; Arianna Collari, Sapienza Università di Roma, Marta Fedeli, Roma ; Ilaria Frumenti, Università di Roma Tre ; Francesca Galanti, Sapienza Università di Roma ; Federico Gallinucci, Sapienza Università di Roma ; JeanPhilippe Goiran, CNRS-Université de Lyon/ UMR Archeorient ; Carole Mabboux, École française de Rome ; Bianca Magliochetti, Sapienza Università di Roma ; Cécile Martini, École française de Rome ; Christian Mazet, École française de Rome ; Milena Mimmo, LabexMed, Centre Camille Jullian ; Ascanio Modena Altieri, Sapienza Università di Roma ; Emanuele Musa, Università di Roma Tre ; Francesco Perotta, Sapienza Università di Roma ; Charles-Edouard Sauvin, Centre des Monuments Nationaux ; Paolo Tomassini, École française de Rome ; Emmanuel Turquin, École française de Rome.

Partenariats institutionnels : Parco Archeologico di Ostia Antica

Établissement éditeur : EFR

Établissements porteurs de l'opération : EFR

Chroniques de l'EFR

https://journals.openedition.org/cefr/3777

https://journals.openedition.org/cefr/2154 


\section{Introduction}

1 L'étude du môle nord-sud de Portus, volet archéologique du programme quinquennal de l'EFR "Ostie-Portus, hub de l'Empire romain", s'est concentrée lors des deux premières campagnes de 2017 et $2018^{1}$ sur les portions terminales de la structure (fig. 1, ABC). Après une année d'interruption des opérations de terrain en 2019, la campagne $2020^{2}$, réalisée en collaboration avec le Parco Archeologico di Ostia Antica (Concession de fouille $\mathrm{n}^{\circ} 1855$ du 05/05/2020), s'est en revanche concentrée sur la partie initiale du môle située dans l'angle nord-ouest des entrepôts dits de Trajan, dans le prolongement de la colonnade du dit Portique de Claude (fig. 1, D)

Fig. 1. Localisation des différents secteurs de fouille et des nouveaux carottages réalisés en 2020 sur le môle nord-sud de Portus.

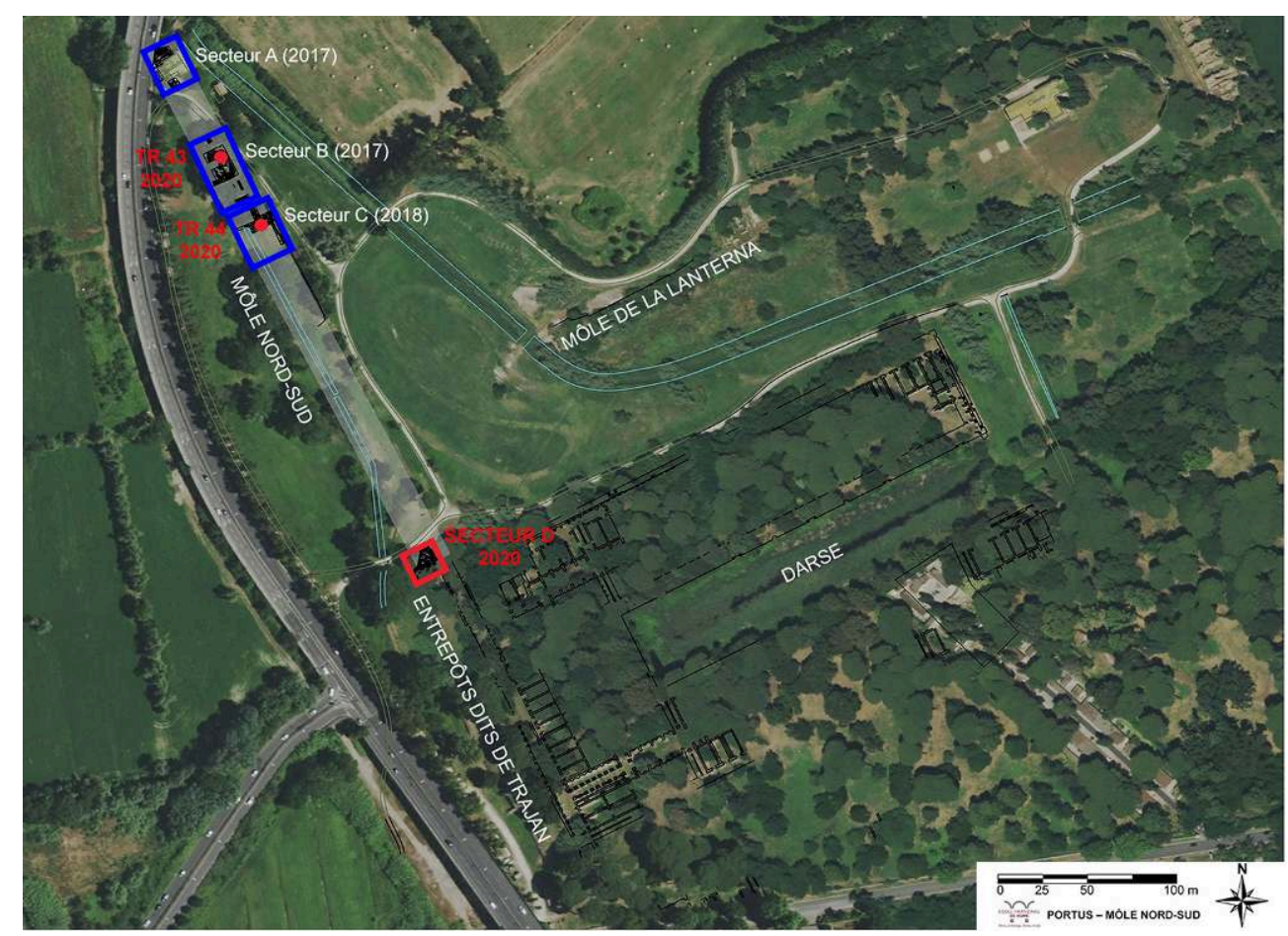

( $\mathrm{EFR} / \mathrm{M}$. Mimmo, I Frumenti.

2 Cette zone a la particularité d'être composée de deux parties distinctes : la partie ouest, surélevée, comprend ce qui reste de la structure tardive du môle; la partie est, visible directement sur le niveau de circulation actuel, comprend en particulier les restes affleurant d'un modeste édifice thermal, également daté de l'Antiquité tardive, qui a probablement été inséré directement sur les structures du môle original de l'époque claudienne.

\section{Objectifs de la campagne 2020}

3 Les objectifs initiaux étaient d'enquêter sur toute la partie initiale du môle, y compris la zone thermale, mais au cours des phases d'organisation de la campagne au printemps 2020, les mesures sanitaires anti-Covid19 se durcissant, il a été décidé de limiter 
l'enquête de terrain prévue en septembre à la seule zone de la structure tardive du môle (fig. 2). En effet, la participation des collègues résidents en France devenant compromise, nous avons préféré adapter nos objectifs autour d'une équipe réduite aux seuls résidents en Italie, reportant à septembre 2021 la fouille du reste du secteur.

Fig. 2. Vue générale de la structure tardive du môle (USM 22).

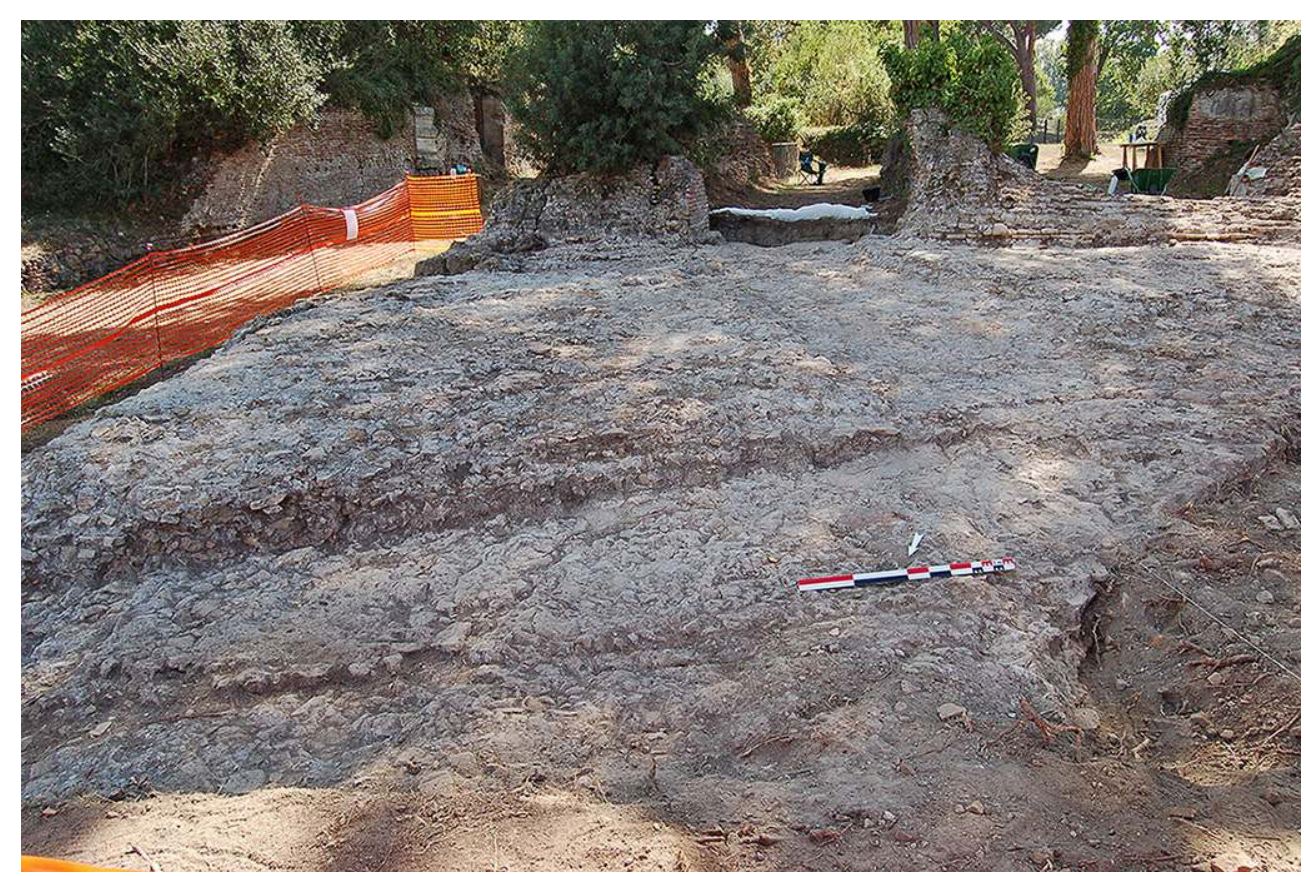

(B) EFR/J. Cocquyt.

$4 \mathrm{Au}$ cours de cette campagne 2020, plus de $70 \mathrm{~m}^{2}$ de structure ont été dégagés entre la zone du môle tardif et le vaste complexe architectural tardo-antique qui s'y lie du côté sud. Ce dernier, communément appelé Antemurale, se présente comme un prolongement des entrepôts dits de Trajan et coure sur une largeur d'environ $16 \mathrm{~m}$ tout le long de l'ensemble du dit Portique de Claude, couvrant ainsi la façade maritime des entrepôts.

\section{Données de terrain}

5 À la différence des deux premières campagnes, les structures mises au jour en 2020 concernent exclusivement l'Antiquité tardive et aucune structure impériale n'a été identifiée sur l'ensemble du secteur fouillé. Pour les commodités d'enregistrement, ce secteur D a été divisé en 6 sondages (fig. 3). 
Fig. 3. Planimétrie détaillée du secteur $D$.

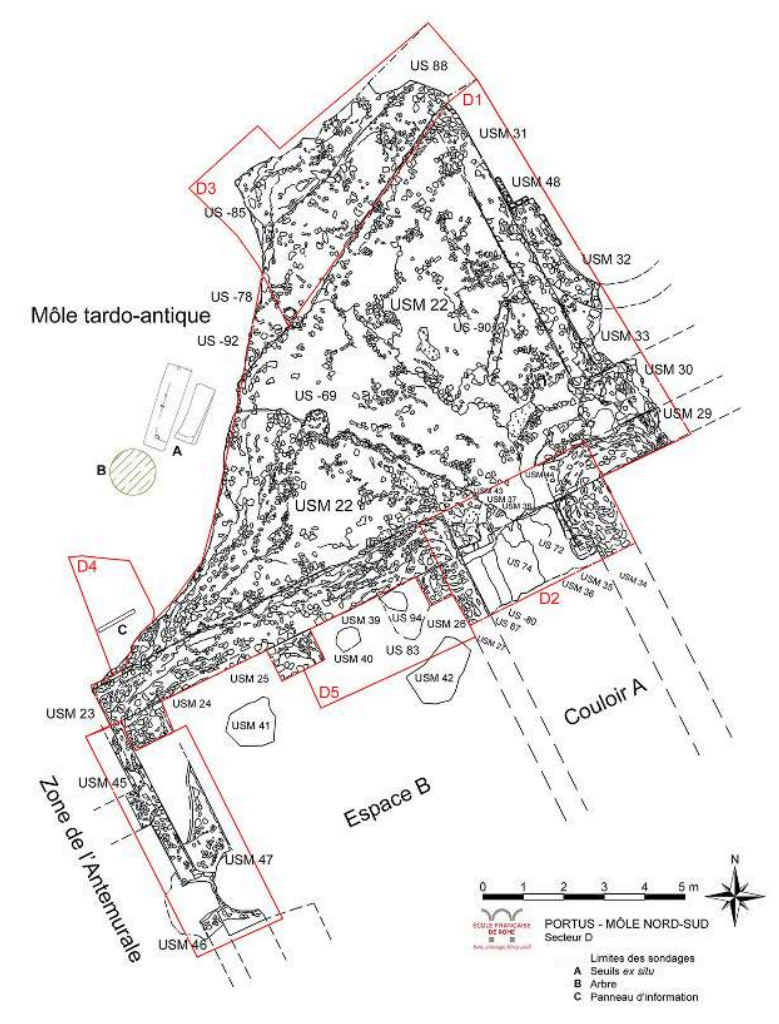

( EFR/M. Mimmo, I. Frumenti, P. Tomassini.

\section{Sondage D1}

6 Le sondage D1 est le plus vaste et comprend l'ensemble de ce qui reste de la partie tardo-antique du môle (USM 22) contre laquelle l'édifice thermal s'insère postérieurement (USM 31, USM 32, USM 33, USM 48), au-dessus du niveau original du môle de Claude. Cette dernière donnée n'est pour l'instant qu'hypothétique et devra être vérifiée lors de la prochaine campagne.

7 Sont également inclus dans le sondage D1 l'élévation conservée du large mur qui ferme au nord le complexe architectural de l'Antemurale (USM 23, USM 29) ainsi que les piliers USM 24, USM 25 et USM 26 qui, avec l'USM 35 du sondage D2, devaient supporter les arcs de soutien du chemin de ronde de l'enceinte tardo-antique construite contre la face interne du mur nord de l'Antemurale.

\section{Sondage D2}

Le sondage D2 (fig. 4) s'est concentré sur la zone de passage créée postérieurement à la construction du mur nord de l'Antemurale (USM 23 et USM 29) afin de permettre la communication directe entre la zone du môle tardo-antique et le couloir A. Délimité par les murs USM 27 et USM 34, ce couloir semble avoir été organisé en même temps que le passage pour servir de rampe d'accès entre le niveau surélevé du môle et le niveau de circulation dans l'Antemurale, estimé à environ $2 \mathrm{~m}$ en dessous. Les US 72 et 74 correspondent aux deux dernières couches de béton de tuileau du niveau de circulation de ce long couloir-rampe qui devait s'étendre sur près de 20 mètres. 
Fig. 4. Vue du sondage D2 en fin de fouille.

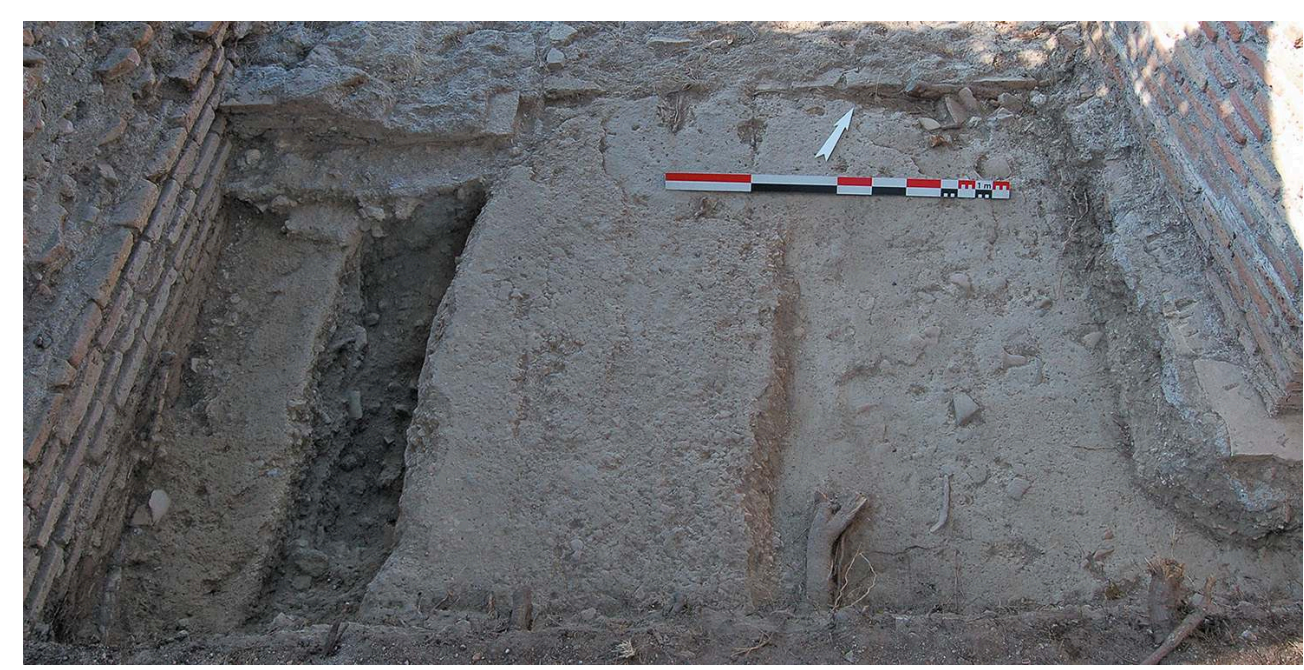

(B) EFR/J. Cocquyt.

\section{Sondage D3}

Le sondage D3 correspond à l'élargissement vers le nord du sondage D1, fouillé précédemment. Le but était de repousser au maximum la limite de fouille afin d'identifier la limite nord de la profonde taille dont ce môle a fait l'objet, sans doute à un stade avancé de la vie du port, afin de permettre un passage plus direct des bateaux qui voulaient y pénétrer. La trop grande proximité du chemin de terre du parcours de visite du site ne nous a pas permis d'identifier précisément cette limite mais des indices, notamment altimétriques, nous font penser que cette dernière doit être voisine.

\section{Sondage D4}

Le sondage $\mathrm{D} 4$ a été ouvert de façon très éphémère, le temps de démonter l'ancien panneau du parcours de visite devenu inutile. Ce sondage a permis également de vérifier la continuité structurelle entre la structure du môle tardo-antique et la fondation du complexe de l'Antemurale.

\section{Sondage D5}

11 Le sondage D5 visait à déterminer le niveau de fondation des piliers USM 25 et USM 26 afin de le comparer à celui d'un autre pilier de cette même phase de construction présent dans le couloir A (USM 35 du sondage D2). Après avoir dégagé plusieurs éléments des arcs en briques effondrés que soutenaient ces piliers, la fouille, devenue trop profonde, a été interrompue pour raison de sécurité, sans pouvoir atteindre leur fondation, sans doute située en lien avec le niveau de circulation de l'espace B apparemment bien plus bas que celui du couloir A, tandis que le pilier USM $35 \mathrm{du}$ sondage D2 s'appuie directement sur un des premiers sols en béton de tuileau de la rampe du couloir A (US 72). 


\section{Sondage D6}

12 Le sondage D6 s'est limité à un bref nettoyage de surface le long de l'angle interne ouest de l'espace B afin de rendre plus visibles les relations stratigraphiques entre les différentes structures conservées dans cette zone et, en particulier, le lien entre le mur périmétrique de l'Antemurale (USM 45) et la structure de l'abside USM 47.

\section{Premières interprétations des résultats}

L'observation des relations stratigraphiques entre les structures, les premières estimations chronologiques du mobilier recueilli et notre connaissance préalable des techniques de construction à Portus nous permettent de reconnaître, à titre provisoire, cinq phases de construction dans la zone du secteur fouillé en 2020 (fig. 5 et 6).

Fig. 5. Élévation et coupe est-ouest du sondage D, vue du nord.
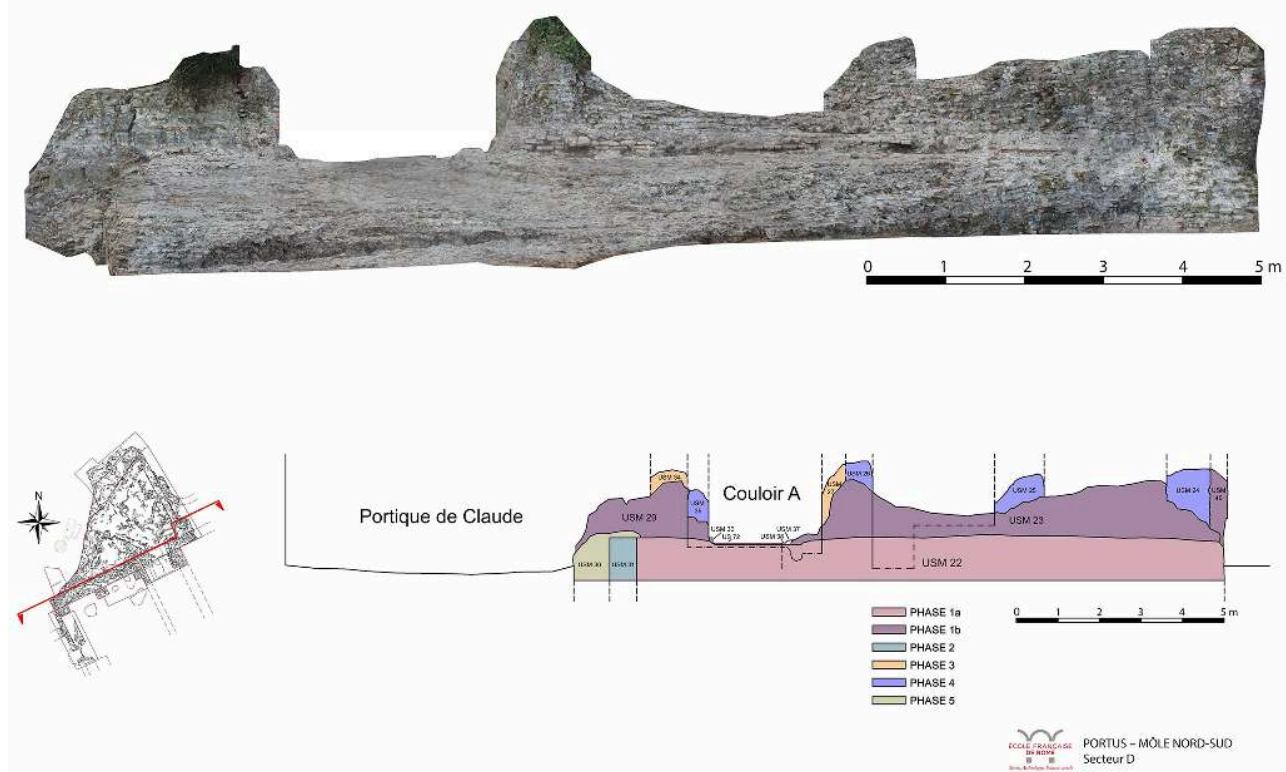

(B) EFR/M. Mimmo, I. Frumenti. 
Fig. 6. Planimétrie des principales phases identifiées dans le secteur D.

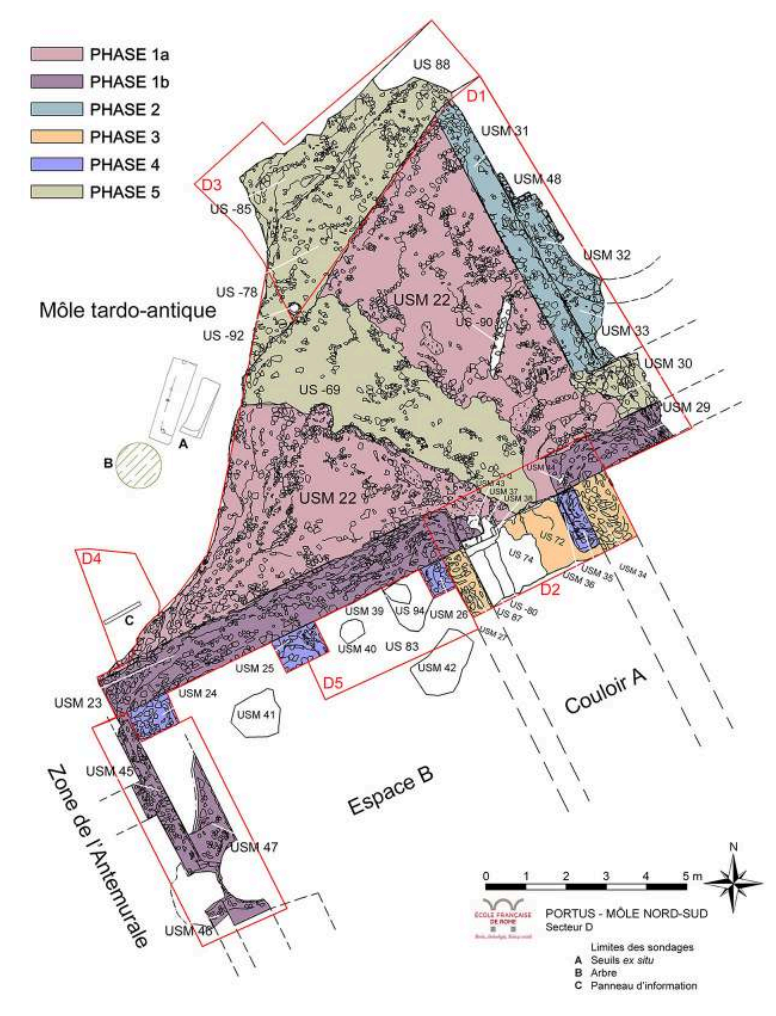

( $\mathrm{EFR/M}$. Mimmo, I. Frumenti.

14 La phase 1a correspond au chantier de construction du môle tardo-antique, contemporain des fondations du complexe de l'Antemurale. La phase $1 \mathrm{~b}$ concerne l'élévation des murs périmétriques de ce complexe.

La phase 2, sans doute de peu plus tardive, correspond à l'installation de l'édifice thermal qui sera l'objet central de la campagne 2021.

La phase 3, peut-être consécutive à la précédente, correspond à l'ouverture dans le mur nord de l'Antemurale, concomitante à la construction du couloir A qui, grâce à la longue rampe organisée sur toute sa largeur, permettait d'accéder commodément du môle aux espaces du rez-de-chaussée de l'Antemurale, comme l'espace B.

17 La phase 4 correspond aux traces de l'enceinte tardo-antique de Portus, traditionnellement datés du dernier quart $d u v^{e}$ siècle, et concerne en particulier une série de piliers qui soutenaient, dans cet angle stratégique de la muraille, son chemin de ronde.

La phase 5 regroupe plusieurs traces d'aménagements vraisemblablement postérieurs à l'Antiquité tardive dont la plus importante correspond à la taille en entonnoir du môle tardo-antique et l'aménagement d'un parcours en pente qui, de la façade portuaire du môle permettait d'accéder au couloir $\mathrm{A}$ et donc à la zone de l'Antemurale. Cette cassure volontaire du môle, bien visible sur plusieurs gravures et plans anciens de Portus, est probablement à mettre en relation avec la création d'un nouveau passage pour les bateaux qui voulaient pénétrer directement dans le port interne et accéder aux entrepôts sans avoir à contourner toute la longueur du môle nord-sud et traverser la partie centrale du bassin de Claude qui à l'époque avait sans doute déjà commencé à s'envaser. 


\section{Réalisation de deux nouveaux carottages sur le môle}

'implication de nos travaux comme de ceux des différentes recherches internationales à Portus dans le cadre du projet FOSPHORA (Fos-Ostie-Portus. Harbours of Roman Antiquity ) financé par A*Midex, Initiative d'excellence de l'Université Aix-Marseille ${ }^{3}$, a permis la réalisation de deux nouveaux carottages dans les structures du môle nord-sud ; le premier (fig. 1, TR 44) dans la portion terminale du môle de Claude et le second (fig. 1, TR 43) dans la portion terminale de son extension sévérienne.

L'analyse de ces échantillons par l'équipe de Jean-Philippe Goiran et Stoil Chapkanski est toujours en cours au moment de la rédaction de cette contribution et nous attendons d'importants résultats permettant de préciser nos connaissances sur les techniques de construction des infrastructures portuaires romaines ${ }^{4}$, mais les coupes provisoires fournies (fig. 7) nous permettent déjà quelques observations préliminaires.

Fig. 7. Restitution schématique de la stratigraphie des nouveaux carottages TR43 et TR44

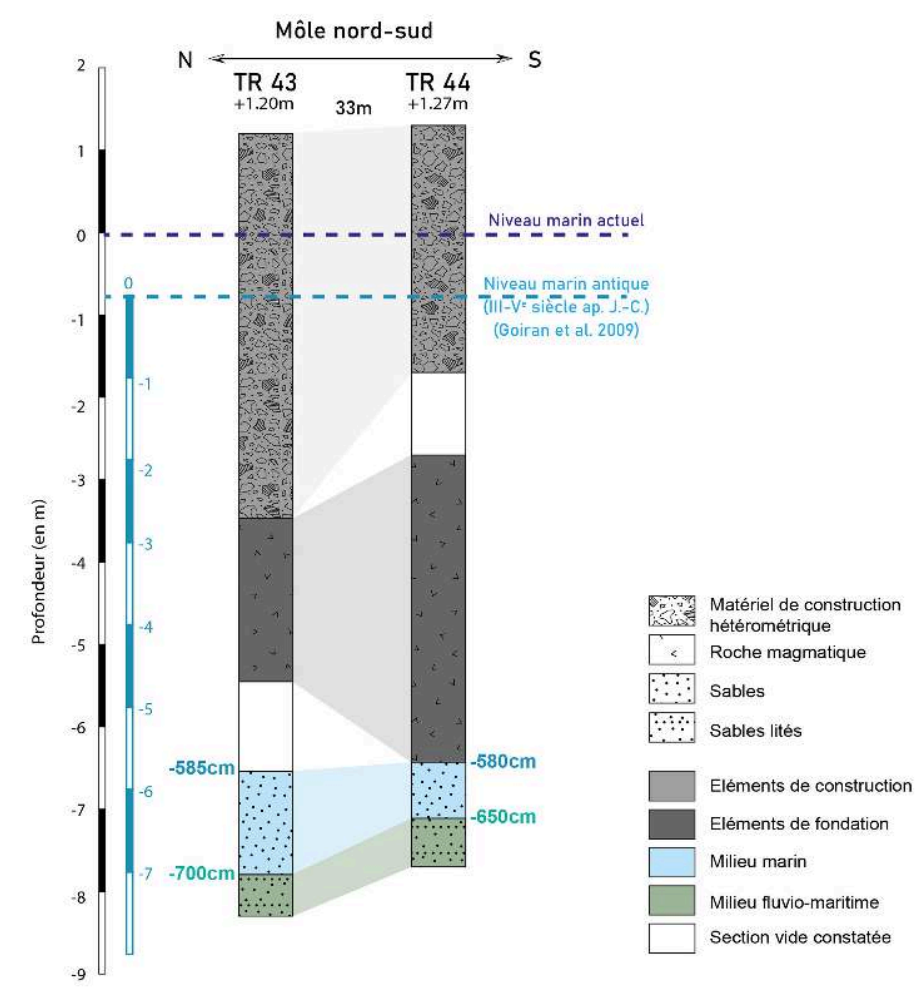

\section{(B) J.-Ph. Goiran, M. Le Doare.}

21 La profondeur de la fondation des deux portions du môle est équivalente autour de 5,80-5,85 m sous le niveau marin antique alors que le niveau supérieur des deux fondations semble différer d'environ 1,8 mètres.

Les étonnantes sections vides constatées sur les deux carottages ne sont pas sans poser des problèmes d'interprétation même s'il semble pour l'instant raisonnable de reconnaitre un système de fondation par immersion de très gros blocs de roche dont l'enchevêtrement anarchique, résultant de leur chute irrégulière depuis la surface située à plus de 5 mètres de hauteur, a dû créer de nombreux espaces vides entre les roches dans lesquels seule l'eau du bassin pouvait pénétrer et que les deux carottages 
de 2021 ont traversé. Cette première tentative d'interprétation permettrait de constater une certaine continuité technologique dans la construction des fondations de môles entre l'époque claudienne et l'époque sévérienne mais nous attendrons les analyses de Stoil Chapkanski qui permettront l'identification précise des matériaux utilisés, pour préciser cette hypothèse très provisoire.

\section{BIBLIOGRAPHIE}

BUKOWIECKI, MIMMO, SAUVIN 2019

E. Bukowiecki, M. Mimmo, C.-E. Sauvin, « Portus. Le môle nord-sud de Portus. Seconde campagne de fouille (2018) », Chroniques des activités archéologiques de l'École française de Rome, en ligne, https://journals.openedition.org/cefr/3777.

BUKOWIECKI, FABRO, MIMMO 2020

E. Bukowiecki, R. Fabro, M. Mimmo, « Portus. Le môle nord-sud de Portus. Première campagne de fouille (2017) », Chroniques des activités archéologiques de l'École française de Rome, en ligne, https:// journals.openedition.org/cefr/2154.

BUKOWIECKI, MIMMO (sous presse)

E. Bukowiecki, M. Mimmo, « Infrastructures portuaires à Portus. Les fouilles récentes de l'École française de Rome ", Actes du XII colloque historique de Fréjus "Les ports dans l'espace méditerranéen antique. Fréjus et les ports maritimes”, Fréjus 16-17 novembre 2018), sous presse.

CHAPKANSKI et al. 2021

S. Chapkanski, J.-P. Goiran, C. Rosa, S. Keay, S. Kay, E. Damien, X. Gallet, « Analyses des matériaux de construction bruts par la Spectroscopie Moyen InfraRouge (SMIR). Application à l'étude du môle nord de Portus, le port antique de Rome et création d'une base minéralogique spectrale » [notice archéologique], Bulletin archéologique des Écoles françaises à l'étranger, en ligne, à paraître.

\section{NOTES}

1. BUKOWIECKI, MIMMO, SAUVIN 2019 ; BUKOWIECKI, FABRO, MIMMO 2020 ; BUKOWIECKI, MIMMO (sous presse).

2. Nous remercions chaleureusement tous ceux qui ont favorisé notre présence à Portus et le bon déroulement de la campagne de fouilles malgré les contraintes imposées par les protocoles sanitaires anti-Covid19. Outre les participants pour leur enthousiasme et leur professionnalisme, nous tenons à remercier tout particulièrement nos précieux interlocuteurs au Parco Archeologico di Ostia Antica, Marina Lo Blundo, Cristina Genovese, Claudia Tempesta, Claudia Mornati, Stefania Pietrini et Franca Tortora ainsi que les jardiniers de la société ALES pour leur généreuse aide logistique.

3. Voir https://amidex.hypotheses.org/9931.

4. CHAPKANSKI et al. 2021. 


\section{INDEX}

sujets https://ark.frantiq.fr/ark:/26678/pcrtVemT6o6YVG peuples https://ark.frantiq.fr/ark:/26678/pcrtkERiWybjc4

Thèmes : EFR

Année de l'opération : 2020

chronologie https://ark.frantiq.fr/ark:/26678/pcrtxT02uJOogm, https://ark.frantiq.fr/ark:/ 26678/pcrtof7EHNsS2e, https://ark.frantiq.fr/ark:/26678/pcrtZTmusVUU24, https:// ark.frantiq.fr/ark:/26678/pcrtIkSWVMVuqB, https://ark.frantiq.fr/ark:/26678/ pcrtH5r3FYBpwe, https://ark.frantiq.fr/ark:/26678/pcrt0auHUwTKix

\section{AUTEURS}

\section{ÉVELYNE BUKOWIECKI}

École française de Rome

\section{ILARIA FRUMENTI}

Università di Roma Tre

\section{JEAN-PHILIPPE GOIRAN}

CNRS-Université de Lyon/ UMR Archeorient

\section{MILENA MIMMO}

Centre Camille Jullian, CNRS-UMR 7299 